\title{
Identification of Patients with Statin Intolerance in a Managed Care Plan: A Comparison of 2 Claims-Based Algorithms
}

\author{
Brandon K. Bellows, PharmD, MS; Amy M. Sainski-Nguyen, PhD; \\ Cody J. Olsen, PharmD, BCPS; Susan H. Boklage, MS, MPH; Scott Charland, PharmD; \\ Matthew P. Mitchell, PharmD, MBA, FAMCP; and Diana I. Brixner, RPh, PhD
}

\begin{abstract}
BACKGROUND: While statins are safe and efficacious, some patients may experience statin intolerance or treatment-limiting adverse events. Identifying patients with statin intolerance may allow optimal management of cardiovascular event risk through other strategies. Recently, an administrative claims data (ACD) algorithm was developed to identify patients with statin intolerance and validated against electronic medical records. However, how this algorithm compared with perceptions of statin intolerance by integrated delivery networks remains largely unknown.
\end{abstract}

OBJECTIVE: To determine the concurrent validity of an algorithm developed by a regional integrated delivery network multidisciplinary panel (MP) and a published ACD algorithm in identifying patients with statin intolerance.

METHODS: The MP consisted of 3 physicians and 2 pharmacists with expertise in cardiology, internal medicine, and formulary management. The MP algorithm used pharmacy and medical claims to identify patients with statin intolerance, classifying them as having statin intolerance if they met any of the following criteria: (a) medical claim for rhabdomyolysis, (b) medical claim for muscle weakness, (c) an outpatient medical claim for creatinine kinase assay, (d) fills for $\geq 2$ different statins excluding dose increases, (e) decrease in statin dose, or (f) discontinuation of a statin with a subsequent fill for a nonstatin lipid-lowering therapy. The validated ACD algorithm identified statin intolerance as absolute intolerance with rhabdomyolysis; absolute intolerance without rhabdomyolysis (i.e., other adverse events); or as dose titration intolerance. Adult patients (aged $\geq 18$ years) from the integrated delivery network with at least 1 prescription fill for a statin between January 1, 2011, and December 31, 2012 (first fil defined the index date) were identified. Patients with $\geq 1$ year pre- and $\geq 2$ years post-index continuous enrollment and no statin prescription fills in the pre-index period were included. The MP and ACD algorithms were applied to the population, and concordance was examined using individual (i.e., sensitivity, specificity, positive predictive value [PPV], and negative predictive value [NPV]) and overall performance measures (i.e., accuracy, Cohen's kappa coefficient, balanced accuracy, F-1 score, and phi coefficient).

RESULTS: After applying the inclusion criteria, 7,490 patients were evaluated for statin intolerance. The mean (SD) age of the population was 51.1 (8.5) years, and $55.7 \%$ were male. The MP and ACD algorithms classified $11.3 \%$ and $5.4 \%$ of patients as having statin intolerance, respectively. The concordance of the MP algorithm was mixed, with negative classification of statin intolerance measures having high concordance (specificity 0.91 , NPV 0.97) and positive classification of statin intolerance measures having poor concordance (sensitivity 0.45 , PPV 0.21 ). Overall performance measures showed mixed agreement between the algorithms.

CONCLUSIONS: Both algorithms used a mix of pharmacy and medical claims and may be useful for organizations interested in identifying patients with statin intolerance. By identifying patients with statin intolerance, organizations may consider a variety of options, including using nonstatin lipid-lowering therapies, to manage cardiovascular event risk in these patients.

\section{J Manag Care Spec Pharm. 2017;23(9):926-34}

Copyright $\odot 2017$, Academy of Managed Care Pharmacy. All rights reserved.

\section{What is already known about this subject}

While statins are generally considered safe, some patients experience statin intolerance, which leads to reduced adherence, statin dose reduction, and statin switching or discontinuation.

Estimates of statin intolerance from clinical and real-world settings range widely, between $5 \%-25 \%$, which may be due to the lack of a universally accepted definition or management strategy to address its occurrence.

\section{What this study adds}

This study convened a multidisciplinary panel (MP) to determine how managed care organizations would define statin intolerance and operationalize algorithms that identify statin intolerance in their populations.

The MP and validated administrative claims data algorithms, both based on a combination of pharmacy and medical claims, classified $11.3 \%$ and $5.4 \%$, respectively, of patients as having statin intolerance in an integrated delivery network.

The joint use of the algorithms may be useful for all health care delivery organizations (e.g., integrated delivery network, pharmacy benefit managers, and accountable care organizations) in identifying patients with statin intolerance to help reduce cardiovascular event risk in these patients.

工 $\mathrm{n}$ 2013, the guideline from the American College of Cardiology and American Heart Association (ACC/AHA) recommended the use of HMG-CoA reductase inhibitors (statins) to treat blood cholesterol and reduce the risk of cardiovascular events. ${ }^{1}$ This guideline shifted from the traditional "treat-to-target" approach for management of lowdensity lipoprotein cholesterol (LDL-C) and instead focused on the importance of statin use. The guideline recommended a patient-centric focus based on factors such as age, diagnosis 
of atherosclerotic cardiovascular disease (ASCVD), LDL-C laboratory values, comorbidities and cardiovascular event risk, and corresponding treatment with an appropriate intensity of statins. ${ }^{1}$ Because evidence demonstrates that low adherence to or discontinuation of statins because of lack of efficacy or intolerance leads to increased mortality and cardiovascular events, ${ }^{2-5}$ the guideline also stresses the importance of adherence to statins. ${ }^{1}$

While statins are generally considered safe, some patients may experience statin intolerance or intolerable adverse events (e.g., myalgia and/or myopathy), leading to reduced adherence, statin dose reduction, and statin switching or discontinuation. ${ }^{6-8}$ Guidelines and consensus statements generally define statin intolerance as adverse effects attributed by the patient or provider to statins that prevent long-term use and are eliminated when statins are discontinued. ${ }^{9,10}$ Statin intolerance may be characterized as complete (i.e., intolerant to any dose of statins) or partial (i.e., intolerant to some statins or doses) and should not be considered a result of drug-drug interactions or other factors, such as age or hypothyroidism..$^{9,10}$

In clinical trials, approximately $6 \%$ of patients discontinued statins because of statin-associated adverse effects, but this varied depending on the type of statin evaluated. ${ }^{7,8}$ However, the clinical trial estimates may be lower than those seen in real-world settings, since many trials excluded patients with previous adverse events while on a statin and patients at risk of adverse events (e.g., older adults and those with comorbidities). Studies in real-world settings have estimated that 5\%-25\% of patients may experience muscle-related symptoms or statin intolerance. ${ }^{11-13}$

For patients with statin intolerance, health care payers and providers must balance the risks and benefits of continuing statin therapy, reducing the statin dose and/or frequency, or initiating alternative lipid-lowering treatments to mitigate the ongoing risk of cardiovascular events. Furthermore, the current ACC/AHA guideline states that clinicians "may consider the addition of a nonstatin cholesterol-lowering therapy" in patients who are completely statin intolerant. ${ }^{1}$ Identifying and budgeting for patients with statin intolerance, who are potential users of costly specialty medications, may be another tool for health delivery organizations. ${ }^{14}$

Although the clinical guideline and panels have discussed statin intolerance, there is no universally accepted definition or management strategy to address its occurrence.,10,15-18 Identification of patients with statin intolerance within the health care delivery system may be difficult, since documentation of statin intolerance components may not be present in accessible structured administrative data. ${ }^{19}$ In an effort to address this problem, algorithms have recently been developed to identify patients with statin intolerance using data accessible to health care delivery organizations ${ }^{20}$ However, given the uncertainty regarding the definition of statin intolerance, it is unknown how these organizations would define statin intolerance and operationalize such algorithms in their populations.

To address this knowledge gap, the application of 2 algorithms was evaluated in this study: (1) an algorithm developed by a multidisciplinary panel (MP) consisting of regional clinical and integrated delivery network professionals to identify patients with statin intolerance and (2) a previously published statin intolerance algorithm based on administrative claims data (ACD) and validated against electronic medical records from the Henry Ford Health System..$^{20}$ The concurrent validity of the MP algorithm was evaluated against the ACD algorithm within an integrated delivery network database.

\section{Methods}

\section{Study Population and Data Source}

This was a retrospective cohort study examining statin intolerance among patients enrolled in the SelectHealth commercial health plan between January 1, 2010, and December 31, 2014. SelectHealth is a nonprofit, regional managed care organization with commercial, Medicare, and Medicaid plans available that covers over 850,000 lives from the Intermountain Region of the United States. SelectHealth is also part of an integrated delivery network with Intermountain Healthcare, a nonprofit health system with 22 hospitals and over 185 clinics across Utah and Idaho.

All patient data for this study were obtained from SelectHealth's commercial medical and pharmacy claims. Patients were included in this study if they had at least 1 statin fill between January 1, 2011, and December 31, 2012, with the date of the first statin fill in that period defining the index date. Patients were also required to be aged $\geq 18$ years on the index date and have continuous enrollment (defined as no gaps $>90$ days) for at least 1 year before and 2 years after the index date. Only incident statin users during the study period were included (defined as having no statin claims for 1 year before the index statin). Comorbidities of interest and medication use were identified in the pre-index period. Prescription drug use was defined by the presence of a pharmacy claim for the drug in the year before the index statin. This study was reviewed and approved by the Intermountain Healthcare Institutional Review Board.

\section{MP Algorithm}

The MP included 3 physicians and 2 pharmacists from SelectHealth and Intermountain Healthcare with expertise in cardiology, internal medicine, managed care, and formulary management. The MP physicians had clinical experience in managing patients with statin intolerance. The panel was convened in November 2015 and its remit was to postulate an algorithm that could be operationalized by managed care organizations to identify patients with statin intolerance based on pharmacy and medical claims data. 


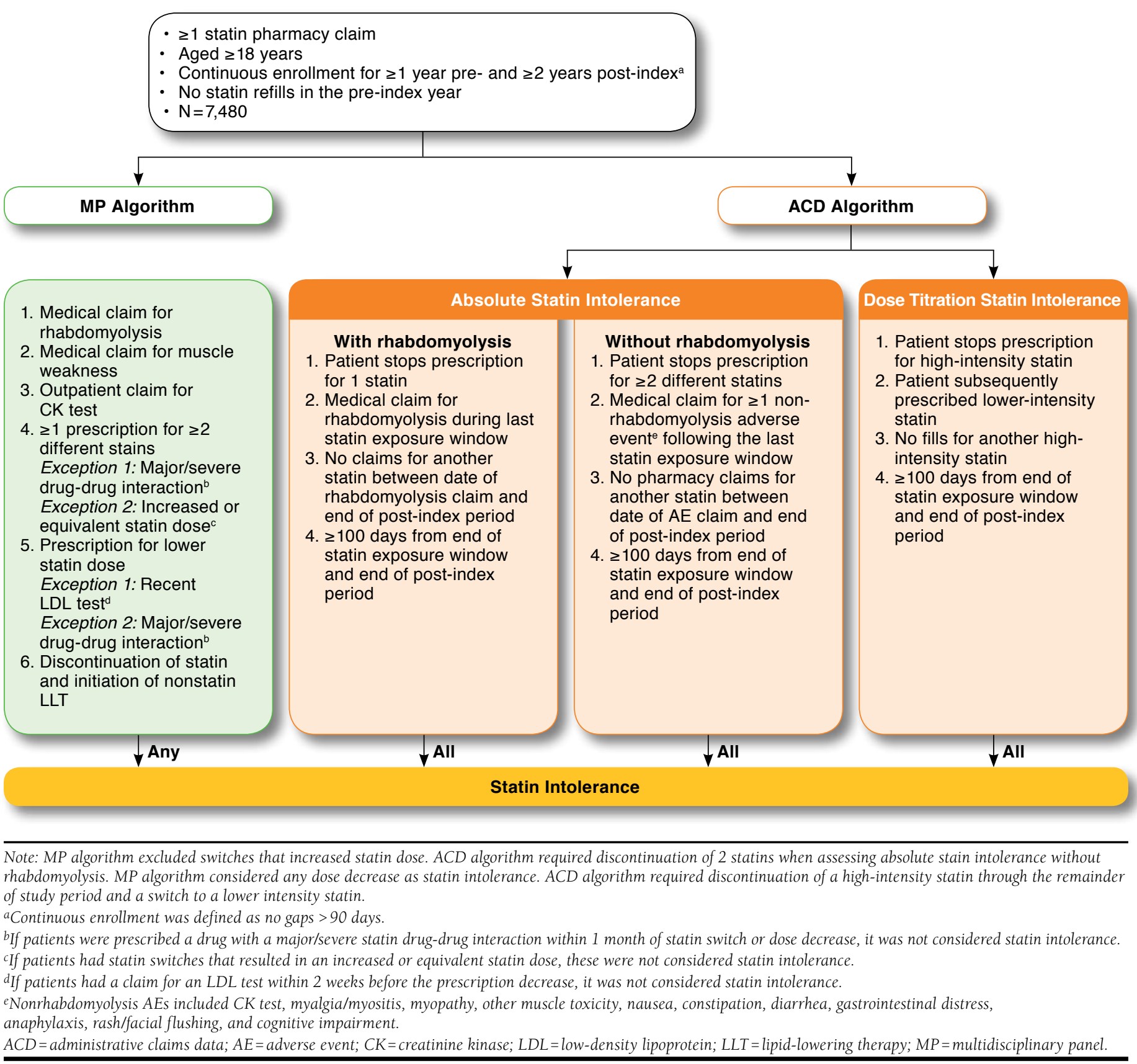

The panel was presented with information on the benefits of statin treatment, various reasons for statin discontinuation, estimated prevalence of statin intolerance, and how statin intolerance is addressed in guidelines and consensus statements. ${ }^{1,9,10,21}$ After receiving this background information, the panel was presented with several examples of algorithms developed from pharmacy and medical claims in other disease states. The panel was then asked to recommend an algorithm to identify patients with statin intolerance in a managed care setting. The panel unanimously defined criteria for statin intolerance and agreed that an algorithm using the combination of pharmacy and medical claims data would be more useful than either one alone. International Classification of Diseases, Ninth Revision, Clinical Modification (ICD-9-CM) 


\section{FIGURE 2 Patient Identification Flowchart}

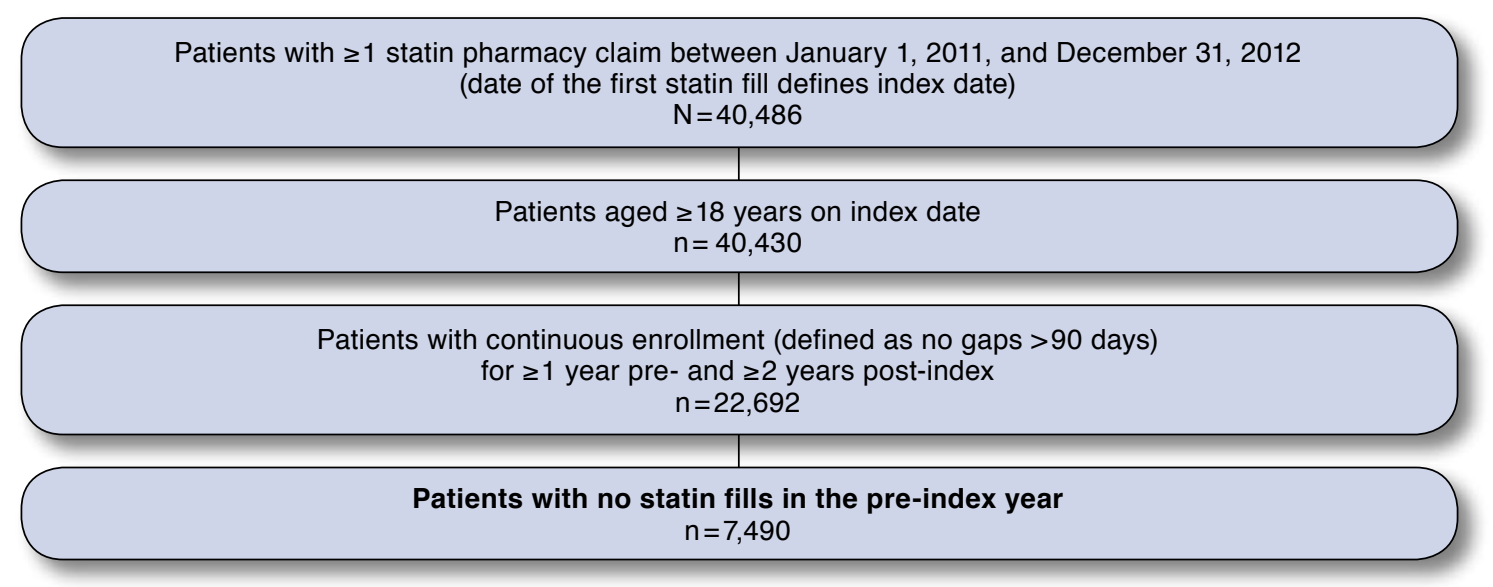

and Healthcare Common Procedure Coding System (HCPCS) codes were used to identify medical claims (Appendix A, available in the online article, includes a complete list of ICD-9-CM and HCPCS codes used).

The panel agreed that statin intolerance is most likely to occur shortly after starting a statin and therefore focused the algorithm on identifying statin intolerance events in patients during the first year after starting a statin (Figure 1). The MP algorithm defined statin intolerance as any of the following 6 criteria occurring after starting a statin: (1) any medical claims for rhabdomyolysis, (2) any medical claims for muscle weakness, (3) any medical claims for creatinine kinase laboratory tests in an outpatient setting, (4) prescription fill for 2 or more statins, (5) prescription fill for a lower dose of the same statin, or (6) discontinuation of a statin, which was defined as no statin fills for at least 6 months after the next expected fill date, with a subsequent fill for a nonstatin lipid-lowering therapy.

While the panel recognized that changes in statin drugs (criterion 4) or dose (criterion 5) would likely be due to statin intolerance, these events may not always be indicative of statin intolerance. Therefore, after considerable discussion, the panel created several exceptions to these criteria. First, it was decided to not consider criteria 4 or 5 to be statin intolerance if patients had a prescription fill for a medication known to have a major/severe or contraindicating drug-drug interaction within 30 days before the event. Drug-drug interactions were derived from drug interaction checking software and were reviewed by a clinical pharmacist for accuracy.22 Second, for criterion 4 , equivalent statin doses were defined (Appendix B, available in online article) and changes in statin therapy were not considered statin intolerance if the alternative statin was the same or increased dose. This was intended to prevent changes to formulary coverage or switches from brand to generic products being classified as statin intolerance. Finally, for criterion 5, decreases in statin dose were not considered statin intolerance if the patient had a claim for an LDL-C laboratory test within 2 weeks before the decrease. This was to allow for potential cholesterol response-guided therapy practiced before the release of the ACC/AHA cholesterol guideline. In addition, for criteria 4 and 5, the panel did not develop any requirements that changes in statin therapy were sustained for the remainder of the follow-up.

\section{ACD Algorithm}

The previously validated ACD algorithm identified 2 types of statin intolerance over 2 years after initiating a statin (Figure 1): (1) absolute statin intolerance, including with and without rhabdomyolysis, and (2) dose titration statin intolerance. To be classified as having absolute statin intolerance with rhabdomyolysis, patients were required to (a) stop filling a prescription for a single statin; (b) have a medical claim for rhabdomyolysis; (c) have no additional prescription fills for a statin after the adverse event (i.e., rhabdomyolysis); and (d) have at least 100 days left in the study period. ${ }^{20}$ Absolute statin intolerance without rhabdomyolysis was similarly defined, except patients were required to stop filling at least 2 different statins, and adverse events other than rhabdomyolysis (e.g., creatinine kinase tests, myalgia/myositis, arthralgia, myopathy, other muscle toxicity, nausea, constipation, diarrhea, other gastrointestinal distress, anaphylaxis, rash/facial flushing, and cognitive impairment) were included.

Dose titration statin intolerance was defined similarly to absolute statin intolerance, except that patients were required to first stop filling a high-intensity statin, subsequently fill a lower intensity statin, and then have no fills for another highintensity statin. 


\section{TABLE 1 Baseline Characteristics of Overall} Population and Patients Identified as Statin Intolerant by Each Algorithm

\begin{tabular}{|c|c|c|c|}
\hline & $\begin{array}{c}\text { Overall } \\
\mathrm{N}=7,490\end{array}$ & $\begin{array}{l}\text { Patients with } \\
\text { SI by MP } \\
\text { Algorithm } \\
n=848\end{array}$ & $\begin{array}{c}\text { Patients with } \\
\text { SI by ACD } \\
\text { Algorithm } \\
n=401\end{array}$ \\
\hline \multicolumn{4}{|c|}{ Demographic characteristics, \% (n) } \\
\hline Age, years, mean (SD) & 51.1 & $(9.4)$ & 51.4 \\
\hline $18-34$ & (459) & (44) & (15) \\
\hline $35-44$ & $18.5(1,385)$ & $16.9 \quad(143)$ & 20.7 \\
\hline $45-54$ & $37.0(2,774)$ & $(295)$ & $(145)$ \\
\hline $55-64$ & $35.0 \quad(2,619)$ & $38.3 \quad(325)$ & (149) \\
\hline $65-74$ & $3.2 \quad(239)$ & (39) & 2.0 \\
\hline $75-84$ & (11) & (2) & 0.2 \\
\hline$\geq 85$ & 0.0 & $(0)$ & 0.0 \\
\hline Male & $55.7 \quad(4,171)$ & $(479)$ & (224) \\
\hline \multicolumn{4}{|c|}{ Clinical characteristics, \% (n) } \\
\hline \multicolumn{4}{|l|}{ Comorbidities } \\
\hline Hyperlipidemia & $69.5(5,202)$ & $(624)$ & $(315)$ \\
\hline Diabetes & $21.9(1,637)$ & $(205)$ & 20.2 \\
\hline Prediabetes & $6.2 \quad(461)$ & (59) & $(22)$ \\
\hline Hypertension & $42.3(3,168)$ & $(409)$ & $(190)$ \\
\hline Metabolic syndrome & (174) & (24) & (13) \\
\hline Overweight & (40) & $(8)$ & 2.0 \\
\hline Obesity & $7.4 \quad(556)$ & $(80)$ & (35) \\
\hline ASCVD & $11.3 \quad(846)$ & (153) & 19.5 \\
\hline $\begin{array}{l}\text { Coronary heart } \\
\text { disease }\end{array}$ & $8.2 \quad(617)$ & $13.8 \quad(117)$ & 16.2 \\
\hline Stroke & $3.2 \quad(238)$ & (49) & (17) \\
\hline $\begin{array}{l}\text { Peripheral vascular } \\
\text { disease }\end{array}$ & (43) & (5) & 1.0 \\
\hline $\begin{array}{l}\text { Chronic kidney } \\
\text { disease }\end{array}$ & $1.9 \quad(142)$ & (21) & 1.5 \\
\hline Hypothyroid & $13.6 \quad(1,018)$ & (132) & 16.0 \\
\hline Cancer & $5.1 \quad(382)$ & $(64)$ & (24) \\
\hline \multicolumn{4}{|l|}{ Drug classes } \\
\hline Antidiabetic & $20.6(1,541)$ & (191) & 20.0 \\
\hline Antihypertensive & $34.4 \quad(2,574)$ & $(310)$ & (146) \\
\hline Antianginal agents & (128) & (34) & (11) \\
\hline Beta blocker & $11.4 \quad(856)$ & (128) & 15.5 \\
\hline $\begin{array}{l}\text { Calcium channel } \\
\text { blocker }\end{array}$ & $7.2 \quad(536)$ & (77) & (36) \\
\hline Diuretic & $11.3 \quad(843)$ & (120) & 11.5 \\
\hline $\begin{array}{l}\text { Thyroid agent } \\
\end{array}$ & $12.6 \quad(944)$ & $14.5 \quad(123)$ & 14.7 \\
\hline $\begin{array}{l}\text { Nonstatin lipid- } \\
\text { lowering therapy }\end{array}$ & $8.6 \quad(645)$ & $12.5 \quad(106)$ & 10.5 \\
\hline
\end{tabular}

$A C D=$ administrative claims data; $A S C V D=$ atherosclerotic cardiovascular disease; $M P=$ multidisciplinary panel; $S D=$ standard deviation; $S I=$ statin intolerance

For absolute statin intolerance, the ACD algorithm also defined statin exposure windows within which the adverse events identified must have occurred. The statin exposure window ended on the expected fill date of each statin prescription fill. However, when filling the same statin and dose, the ACD algorithm allowed for gaps in therapy less than or equal to 30 days in defining statin exposure windows. That is, if a

\section{TABLE 2 Prevalence of Statin Intolerance}

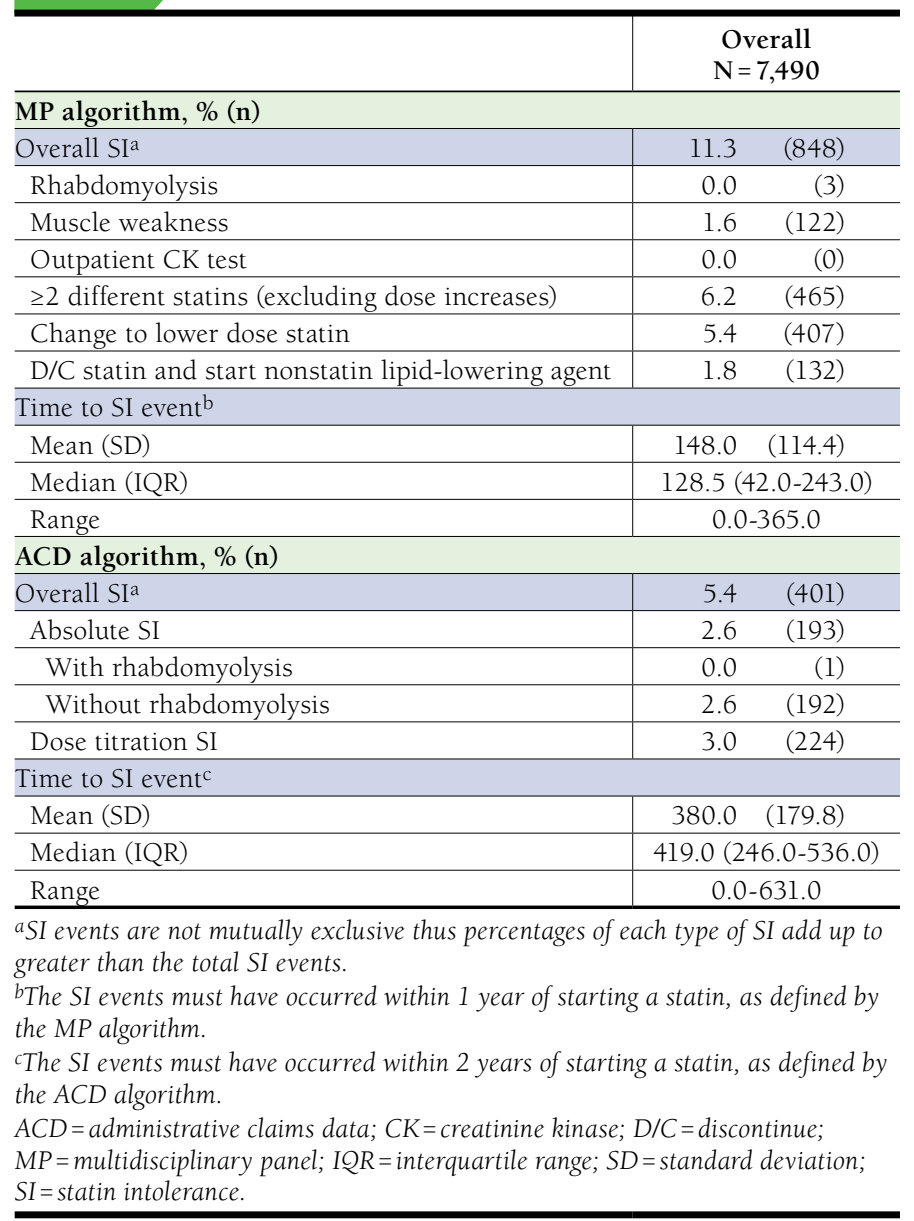

patient filled the same statin prescription within 30 days after the expected fill date of the last prescription, regardless of whether it was a 30- or 90-day fill, it was considered part of the same statin exposure as the previous fill. New statin exposure windows occurred when patients filled the same statin prescription more than 30 days after the expected fill date, filled a prescription for a different statin dose, or switched statins. Adverse events were considered statin intolerance only if they occurred during a statin exposure window.

\section{Analysis}

Descriptive statistics, including mean, standard deviation (SD), frequency, and percentage were reported for baseline demographics (e.g., age and sex) and clinical characteristics (e.g., comorbidities and prescription drug classes used) as appropriate. The statin intolerance classification of patients by each algorithm was reported, and the concordance of the MP algorithm and the ACD algorithm was examined. Individual concordance measures (i.e., those focused on either the positive or negative 


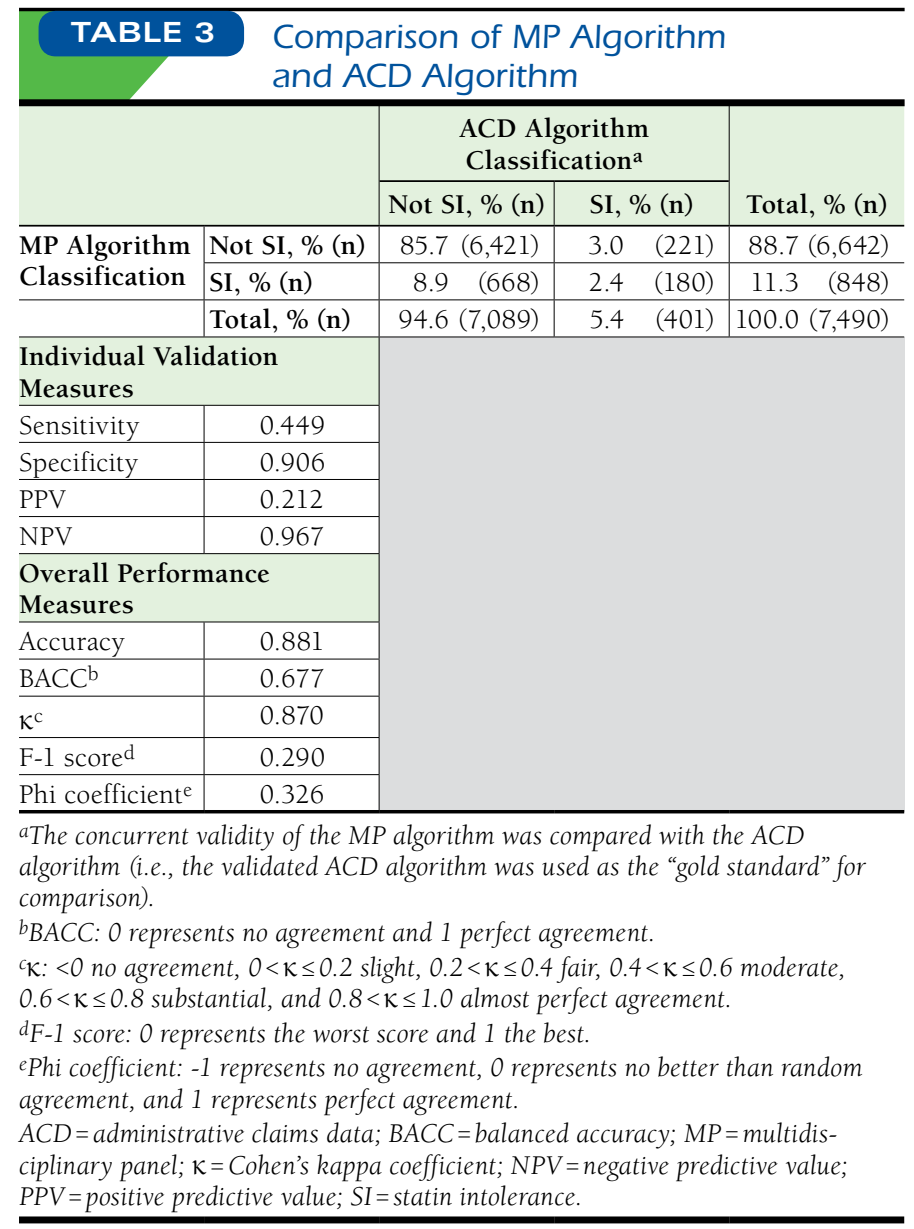

classification, including sensitivity, specificity, positive predictive value $[\mathrm{PPV}]$, and negative predictive value [NPV]) and overall performance measures (i.e., accuracy; Cohen's kappa coefficient $[\kappa]-\kappa<0$ indicate no agreement, whereas $0.8<\kappa \leq 1.0$ almost perfect agreement; balanced accuracy [BACC] -0 is no agreement and 1 perfect agreement; F-1 score-0 represents the worst score and 1 the best; and phi coefficient minus -1 is no agreement, 0 is no better than random agreement, and 1 is perfect agreement) of the MP algorithm were reported because of the expected imbalance of the classification. The imbalance in classifications was expected, since it was anticipated that the large majority of patients would be classified as not having statin intolerance by both algorithms. Thus, measures such as the phi coefficient (also known as Matthews correlation coefficient), which accounts for patients identified with statin intolerance by both algorithms and those not to have statin intolerance by both algorithms and is considered to be a "balanced measure" even if there is a large imbalance in the classifications, were used. ${ }^{23}$ All statistical tests were performed using R, version 3.1 (R Foundation for Statistical Computing, Vienna, Austria).

\section{Results}

After applying the inclusion criteria to the 40,486 patients with at least 1 statin prescription fill, 7,490 patients were newly prescribed statins as defined by the inclusion criteria (Figure 2). The mean (SD) age was 51.1 (8.5) years, and $55.7 \%$ of the population were male (Table 1). In the pre-index period, $69.5 \%$ of the cohort had a diagnosis of hyperlipidemia; $42.3 \%$ had hypertension; and $21.9 \%$ had diabetes. Additionally, $11.3 \%$ had a diagnosis for clinical ASCVD. Further, only 8.6\% of the patients had 1 or more prescriptions for nonstatin lipidlowering agents.

The MP algorithm classified $11.3 \%$ of patients as having statin intolerance (Table 2 ). Since the criteria were not mutually exclusive, some patients met more than 1 criterion. The majority of patients were classified as having statin intolerance by the MP algorithm due to having at least 2 different statins (6.2\%, excluding patients with a switch that resulted in an equivalent or increased statin dose) and changing to a lower dose statin (5.4\%). Applying the ACD algorithm to the study population, $5.4 \%$ of patients were classified as having statin intolerance. Similarly, the ACD algorithm had more patients classified as having dose titration intolerance (3.0\%), but $2.6 \%$ of patients had absolute statin intolerance without rhabdomyolysis. The MP algorithm required statin intolerance events to occur within 1 year of starting a statin and had a mean (SD) time to a statin intolerance event of 148.0 (114.4) days after the index date. The ACD algorithm, however, followed patients for 2 years after starting a statin and had a mean (SD) time to a statin intolerance event of 380.0 (179.8) days.

The characteristics of the patients identified as having statin intolerance by both algorithms are shown in Table 1. Both algorithms had similar proportions of patients with statin intolerance who had a pre-index diagnosis of hyperlipidemia (MP 73.6\% vs. ACD 78.6\%), hypertension (MP 48.2\% vs. ACD 47.4\%), and diabetes (MP 24.2\% vs. ACD 20.2\%). In addition, $18.0 \%$ of patients classified as having statin intolerance by the MP algorithm had ASCVD, and 19.5\% were classified with ASCVD by the ACD algorithm. Nonstatin lipid-lowering therapy use was also similar (MP 12.5\% vs. ACD 10.5\%).

Comparison of patient classification between the algorithms indicated that $88.1 \%$ of classifications were concordant, and $11.9 \%$ were discordant (Table 3). As expected, concordance of the MP algorithm with the ACD algorithm was mixed, being high for negative statin intolerance classification (specificity 0.91 and NPV 0.97) but poor for positive statin intolerance classification (sensitivity 0.45 and PPV 0.21 ). The overall performance measures were also mixed, with Cohen's $\kappa$ showing near perfect agreement (0.87) and accuracy (0.88) and BACC (0.68) showing good agreement; however, the phi coefficient (0.33) and F-1 score (0.29) showed only slightly better than random agreement. The lower agreement for the phi coefficient and F-1 score was expected, since these measures account for 
the large imbalance in the statin intolerance classification (i.e., the majority of patients were classified as not having statin intolerance). ${ }^{23}$

\section{Discussion}

This study assessed the concurrent validity of 2 algorithms based on medical and pharmacy claims data for identification of patients with statin intolerance in a care delivery setting. Of the 2 algorithms evaluated, one was developed by an MP consisting of regional clinical and managed care experts. This algorithm was evaluated against a previously developed ACD algorithm that was validated using electronic medical records. ${ }^{20}$ This study found that among patients initiating treatment with a statin, the MP algorithm classified $11.3 \%$ as having statin intolerance, whereas the ACD algorithm classified only $5.4 \%$, both of which fall within estimates from observational data. ${ }^{11,12}$ The concordance of the MP algorithm versus the ACD algorithm was mixed. While it had high specificity, NPV, and Cohen's $\kappa$, it had poor sensitivity, PPV, and a phi coefficient, which may be considered a limitation of this and other algorithms of this nature. Appropriate identification of statin intolerance is critical for health care delivery organizations to optimally manage patients at risk for cardiovascular events. Ultimately, identification of patients with statin intolerance may provide an opportunity for further cardiovascular event risk reduction by providing more individualized management of cholesterol through a patient-centered approach.

A recently published study identifying statin intolerant patients using Medicare claims had similar definitions to the ACD and MP algorithms used in the current study, which may explain the similar statin intolerance prevalence estimates. As in the current study, a decrease in statin dose was one of the largest identifiers of statin intolerance (4.4\% vs. 5.4\% MP and $3.0 \%$ ACD algorithms). However, differences between the results are to be expected given that the current study did not include Medicare patients. ${ }^{24}$

Statins have been shown to decrease cardiovascular events in patients with elevated LDL-C and be cost-effective in patients with a wide range of cardiovascular risk factors. ${ }^{6,25-27}$ However, despite the abundance of evidence demonstrating the benefits of statins, adherence to statin treatment decreases over time and remains poor (approximately 25\% of patients are considered adherent at 5 years after initiation), ${ }^{28,29}$ and up to $25 \%$ of patients experience muscle-related symptoms or statin intolerance. ${ }^{7,11,12}$ For patients with poor adherence, health care systems and providers can undertake efforts to improve statin adherence. Still, cardiovascular risk management of patients with statin intolerance remains difficult and approaches may include increased consultation with the patient, rechallenging statins, use of suboptimal statin doses, alternative statin dosing regimens, and/or use of nonstatin lipid-lowering alternatives. ${ }^{1,30}$
The algorithms used in this study had many similarities. Both used prescription fill patterns and adverse events to identify statin intolerance events. However, several important differences between the algorithms may explain the differences between the classifications of statin intolerance. For example, the ACD algorithm considered a wider variety of adverse events than the MP algorithm, which led to a greater number of patients classified as having statin intolerance based on adverse events (1.6\% MP vs. 2.6\% ACD). While members of the panel discussed many of the adverse effects included in the ACD algorithm (e.g., cognitive impairment and myalgia), they ultimately decided it would be too difficult to attribute them as due solely to statins using claims data.

In addition, the ACD algorithm required that adverse events occur within a statin exposure window, while the MP algorithm did not require a temporal relationship between statin use and adverse events. This led to a smaller number of patients with statin intolerance due to rhabdomyolysis with the ACD algorithm (e.g., 3 patients for MP vs. 1 patient for ACD). Finally, another requisite of the ACD algorithm was at least 100 days to ensure adequate follow-up time for assessment of statin-taking behavior. The MP algorithm did not require this, except that a statin must have been discontinued for at least 6 months when a nonstatin was started. This likely led to a higher estimate of statin intolerance by the MP algorithm.

While there were several differences between the MP and ACD algorithms, both may be useful to a variety of health care delivery organizations. Since the MP algorithm required a shorter duration of follow-up, it may be more applicable to health care delivery organizations when only short term followup data are available. Both algorithms relied on a combination of pharmacy and medical claims, but the majority of statin intolerant patients were identified through pharmacy claims, particularly with the MP algorithm. The pharmacy portions of the algorithms therefore may be used by pharmacy benefit managers to identify statin intolerance when only pharmacy claims data are available.

In the development of the MP algorithm, the panel noted that one of biggest challenges that health delivery organizations will face in identifying statin intolerance using claims is provider coding habits. Providers may not focus on coding adverse events and may relegate adverse events and other patient concerns to text notes, which may or may not be submitted as billable codes and thus may or may not be available to payers. In addition, it was noted that often only 3 or 4 codes may be documented from a visit even if the provider identifies more medical issues. This may also be compounded by patients' openness with their providers. Thus, the panel felt that pharmacy claims may be a more reliable way to identify statin intolerance, although claims data may be potentially confounded by patient adherence and persistence to therapy. The panel also indicated that some of the challenges may 
have arisen because of the switch to ICD-10-CM codes in October 2015 (see Appendix A for equivalent ICD-10-CM and ICD-9-CM codes used in this study) and discussed a learning delay for providers and medical coders. Finally, the panel discussed the difficulty in capturing the use of drug holidays (i.e., a short-term discontinuation of treatment) by providers to assess and re-evaluate statin tolerance issues.

\section{Limitations}

This study has several limitations worthy of discussion. First, the MP algorithm was not independently validated against medical records. However, the ACD algorithm was based on administrative claims data and validated against medical records (sensitivity 0.78, specificity 0.98, PPV 0.64, NPV 0.99, and Cohen's $\kappa$ 0.66) so serves as an acceptable reference group for the concordance of the MP algorithm. ${ }^{20}$ In addition, the application of the ACD algorithm to the population in this study identified fewer patients with statin intolerance than in the validation study (current study $5.4 \%$ vs. validation study $14.0 \%$ ). In the validation study, the majority of patients with statin intolerance were classified as having titration intolerance (11.8\%). This study was performed in a commercially insured population in the Intermountain Region, and the differences seen in statin intolerance incidence may be a result of differences in patient and provider practices between the populations. In this study, only $5.4 \%$ of patients decreased to a lower statin dose. Future studies are warranted to examine the statin prescription fill patterns in larger, more nationally representative populations.

Second, the algorithms examined statin intolerance over different lengths of follow-up (MP 1 year vs. ACD 2 years). This led to a greater time period where patients may have been identified as having statin intolerance by the ACD algorithm, and the MP algorithm may have missed some statin intolerance cases. However, the stricter follow-up time requirements of the ACD algorithm likely led to the overall lower statin intolerance estimates.

Third, the MP algorithm did not require that patients switching statins or decreasing their statin dose be followed to ensure that this change was sustained. This may have caused the algorithm to be susceptible to temporary changes in therapy not indicative of statin intolerance. While the follow-up times could have been varied to make them more equivalent, the authors wanted the MP to develop its algorithm in isolation of the ACD algorithm in order to examine how managed care and clinical decision makers may identify statin intolerance in claims data. Thus, in order to preserve the way in which the algorithms were developed, neither algorithm was altered in the assessment of the concurrent validity.

Finally, emerging evidence of the effect of the release of the 2013 ACC/AHA guideline on statin treatment patterns in patients shows that patients are still undertreated with highintensity statins. ${ }^{31-33}$ This study used a historical dataset that may not reflect current statin treatment patterns, as well as the incidence of statin intolerance with the increased focus on high-intensity statins. Future research should examine the application of statin intolerance identification algorithms using contemporary data. However, panel members discussed their perceptions regarding slow adoption of practice guidelines by health care practitioners and determined that statin intolerance algorithms should also incorporate features consistent with historical treatment patterns.

\section{Conclusions}

The MP and ACD algorithms had mixed concordance, with high scores related to negative statin intolerance classification but relatively poor scores for those associated with positive identification of statin intolerance. Both algorithms used a combination of pharmacy and medical claims and may be useful for all health care delivery organizations (e.g., integrated delivery networks and accountable care organizations) in identifying patients with statin intolerance. In addition, given the proportion of patients classified as statin intolerant via prescription fill patterns, both algorithms may be useful for pharmacy benefit managers as well. By identifying patients with statin intolerance, health care delivery systems may use population health management strategies to help reduce the risk of cardiovascular events in these patients.

\section{Authors}

BRANDON K. BELLOWS, PharmD, MS, Department of

Pharmacotherapy, University of Utah College of Pharmacy, Salt

Lake City, and SelectHealth, Murray, Utah. AMY M. SAINSKI-

NGUYEN, PhD, and DIANA I. BRIXNER, RPh, PhD, Department

of Pharmacotherapy, University of Utah College of Pharmacy,

Salt Lake City. CODY J. OLSEN, PharmD, BCPS, and MATTHEW

P. MITCHELL, PharmD, MBA, FAMCP, SelectHealth,

Murray, Utah. SUSAN H. BOKLAGE, MS, MPH, Regeneron

Pharmaceuticals, Tarrytown, New York, and SCOTT CHARLAND,

PharmD, Sanofi, Bridgewater, New Jersey.

AUTHOR CORRESPONDENCE: Brandon K. Bellows, PharmD, MS, Dept. of Pharmacotherapy, University of Utah College of Pharmacy, 30 S. 2000 E., Rm. 4963, Salt Lake City, UT 84112.

Tel.: 801.587.9639; E-mail: brandon.bellows@pharm.utah.edu.

\section{DISCLOSURES}

This study was funded by Regeneron Pharmaceuticals and Sanofi US. Boklage is employed by, and owns stock in, Regeneron, and Charland is employed by Sanofi. Bellows has received fees from Avenir for advisory board membership and grants from Myriad Genetics, Biogen, Janssen, and National Institutes of Health. Brixner reports advisory board and consultancy fees and grants from Sanofi. Mitchell reports consultancy fees from Sanofi.

Study concept and design were contributed by Bellows, Boklage, Charland, and Brixner. Bellows, Sainski-Nguyen, and Olsen took the lead in data collection, along with Mitchell. Data interpretation was performed by Mitchell, along with the other authors. The manuscript was written by Bellows, Sainski-Nguyen, and Olsen and revised by all the authors. 


\section{ACKNOWLEDGMENTS}

Medical writing support and manuscript preparation was provided by Gauri Saal, Prime, Knutsford, United Kingdom, and funded by Sanofi and Regeneron Pharmaceuticals. The authors thank Eric Cannon, PharmD, FAMCP; Curtis Wander, PharmD, BCPS; Roy Gandolfi, MD; Jeffrey Twitchell, MD; and Frank Yanowitz, MD, FACP, FACC, for their participation in the multidisciplinary panel and development of the statin intolerance algorithm.

\section{REFERENCES}

1. Stone NJ, Robinson JG, Lichtenstein AH, et al. 2013 ACC/AHA guideline on the treatment of blood cholesterol to reduce atherosclerotic cardiovascular risk in adults: a report of the American College of Cardiology/ American Heart Association Task Force on Practice Guidelines. Circulation. 2014;129(25 Suppl 2):S1-45.

2. Ho PM, Magid DJ, Shetterly SM, et al. Medication nonadherence is associated with a broad range of adverse outcomes in patients with coronary artery disease. Am Heart J. 2008;155(4):772-79.

3. Rasmussen JN, Chong A, Alter DA. Relationship between adherence to evidence-based pharmacotherapy and long-term mortality after acute myocardial infarction. JAMA. 2007;297(2):177-86.

4. Wei L, Wang J, Thompson P, Wong S, Struthers AD, MacDonald TM. Adherence to statin treatment and readmission of patients after myocardial infarction: a six year follow up study. Heart. 2002;88(3):229-33.

5. Heeschen C, Hamm CW, Laufs U, et al. Withdrawal of statins increases event rates in patients with acute coronary syndromes. Circulation. 2002;105(12):1446-52

6. Baigent C, Keech A, Kearney PM, et al. Efficacy and safety of cholesterollowering treatment: prospective meta-analysis of data from 90,056 participants in 14 randomised trials of statins. Lancet. 2005;366(9493):1267-78.

7. Kashani A, Phillips CO, Foody JM, et al. Risks associated with statin therapy: a systematic overview of randomized clinical trials. Circulation. 2006;114(25):2788-97.

8. Naci H, Brugts J, Ades T. Comparative tolerability and harms of individual statins: a study-level network meta-analysis of 246955 participants from 135 randomized, controlled trials. Circ Cardiovasc Qual Outcomes. 2013;6(4):390-99.

9. Mancini GB, Tashakkor AY, Baker S, et al. Diagnosis, prevention, and management of statin adverse effects and intolerance: Canadian Working Group Consensus update. Can J Cardiol. 2013;29(12):1553-68.

10. Guyton JR, Bays HE, Grundy SM, Jacobson TA; The National Lipid Association Statin Intolerance Panel. An assessment by the Statin Intolerance Panel: 2014 update. J Clin Lipidol. 2014;8(3 Suppl):S72-S81.

11. Bruckert E, Hayem G, Dejager S, Yau C, Begaud B. Mild to moderate muscular symptoms with high-dosage statin therapy in hyperlipidemic patients—the PRIMO study. Cardiovasc Drugs Ther. 2005;19(6):403-14.

12. Cohen JD, Brinton EA, Ito MK, Jacobson TA. Understanding Statin Use in America and Gaps in Patient Education (USAGE): an internet-based survey of 10,138 current and former statin users. J Clin Lipidol. 2012;6(3):208-15.

13. Graham DJ, Staffa JA, Shatin D, et al. Incidence of hospitalized rhabdomyolysis in patients treated with lipid-lowering drugs. JAMA. 2004:292(21):2585-90

14. Lotvin AM, Shrank WH, Singh SC, Falit BP, Brennan TA. Specialty medications: traditional and novel tools can address rising spending on these costly drugs. Health Aff (Millwood). 2014;33(10):1736-44.
15. Jacobson TA, Ito MK, Maki KC, et al. National lipid association recommendations for patient-centered management of dyslipidemia: part 1-full report. J Clin Lipidol. 2015;9(2):129-69.

16. Jacobson TA, Maki KC, Orringer CE, et al. National Lipid Association recommendations for patient-centered management of dyslipidemia: part 2 J Clin Lipidol. 2015;9(6 Suppl):S1-122.e1.

17. Jellinger PS, Smith DA, Mehta AE, et al. American Association of Clinical Endocrinologists' Guidelines for Management of Dyslipidemia and Prevention of Atherosclerosis. Endocr Pract. 2012;18(Suppl 1):1-78.

18. Banach M, Rizzo M, Toth PP, et al. Statin intolerance - an attempt at a unified definition. Position paper from an International Lipid Expert Panel. Arch Med Sci. 2015;11(1):1-23.

19. Zhang H, Plutzky J, Skentzos S, et al. Discontinuation of statins in routine care settings: a cohort study. Ann Intern Med. 2013;158(7):526-34.

20. Schulman KL, Lamerato LE, Dalal MR, et al. Development and validation of algorithms to identify statin intolerance in a U.S. administrative database. Value Health. 2016;19(6):852-60

21. Jellinger P, Smith D, Mehta A, et al. American Association of Clinical Endocrinologists' Guidelines for Management of Dyslipidemia and Prevention of Atherosclerosis. Endocr Pract. 2012;18(Suppl 1):1-78.

22. Truven Health Analytics. Micromedex Solutions. Online search. 2015. 23. Wei Q, Dunbrack RL Jr. The role of balanced training and testing data sets for binary classifiers in bioinformatics. PLoS One. 2013;8(7):e67863.

24. Colantonio LD, Kent ST, Huang L, et al. Algorithms to identify statin intolerance in Medicare administrative claim data. Cardiovasc Drugs Ther. 2016;30(5):525-33.

25. Mills EJ, Rachlis B, Wu P, Devereaux PJ, Arora P, Perri D. Primary prevention of cardiovascular mortality and events with statin treatments: a network meta-analysis involving more than 65,000 patients. J Am Coll Cardiol. 2008;52(22):1769-81.

26. Heart Protection Study Collaborative Group. Statin cost-effectiveness in the United States for people at different vascular risk levels. Circ Cardiovasc Qual Outcomes. 2009;2(2):65-72.

27. Pandya A, Sy S, Cho S, Weinstein MC, Gaziano TA. Cost-effectiveness of 10-year risk thresholds for initiation of statin therapy for primary prevention of cardiovascular disease. JAMA. 2015;314(2):142-50.

28. Benner JS, Glynn RJ, Mogun H, Neumann PJ, Weinstein MC, Avorn J Long-term persistence in use of statin therapy in elderly patients. JAMA. 2002;288(4):455-61.

29. Choudhry NK, Avorn J, Glynn RJ, et al. Full coverage for preventive medications after myocardial infarction. N Engl J Med. 2011;365(22):2088-97.

30. Ahmad Z. Statin intolerance. Am J Cardiol. 2014;113(10):1765-71.

31. Tran JN, Kao TC, Caglar T, et al. Impact of the 2013 Cholesterol Guideline on patterns of lipid-lowering treatment in patients with atherosclerotic cardiovascular disease or diabetes after 1 year. J Manag Care Spec Pharm. 2016;22(8):901-08. Available at: http://www.jmcp.org/doi/10.18553/ jmcp.2016.22.8.901.

32. Bellows BK, Olsen CJ, Voelker J, Wander C. Antihyperlipidemic medication treatment patterns and statin adherence among patients with ASCVD in a managed care plan after release of the 2013 ACC/AHA Guideline on the Treatment of Blood Cholesterol. J Manag Care Spec Pharm. 2016;22(8):892900. Available at: http://www.jmcp.org/doi/10.18553/jmcp.2016.22.8.892.

33. Lin I, Sung J, Sanchez RJ, et al. Patterns of statin use in a real-world population of patients at high cardiovascular risk. J Manag Care Spec Pharm. 2016;22(6):685-98. Available at: http://www.jmcp.org/doi/full/10.18553/ jmcp.2016.22.6.685. 


\section{APPENDIX A ICD-9-CM and HCPCS Codes Used}

Condition

Statin intolerance-related conditions

Rhabdomyolysis

Muscle weakness

Creatinine kinase tests

Myalgia/myositis

Arthralgia

Myopathy

Other muscle toxicity

Nausea

Constipation

Diarrhea

Other gastrointestinal distress

Anaphylaxis

Rash/facial flushing

Cognitive impairment

Comorbidities

Diabetes

Prediabetes

Hyperlipidemia

Hypertension

Metabolic syndrome

Atherosclerotic cardiovascular disease

Coronary heart disease

Stroke

Peripheral vascular disease

Chronic kidney disease

Overweight

Obesity

Hypothyroid

Cancer

HCPCS = Healthcare Common Procedure Coding System; ICD-9-CM = International Classification of Diseases, Ninth Revision, Clinical Modification.

\section{APPENDIX B Statin Equivalent Doses}

Tier $\quad$ Equivalent Statins

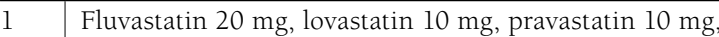
simvastatin $5 \mathrm{mg}$

$2 \quad$ Fluvastatin $40 \mathrm{mg}$, lovastatin $20 \mathrm{mg}$, pitavastatin $1 \mathrm{mg}$, pravastatin $20 \mathrm{mg}$, simvastatin $10 \mathrm{mg}$

$3 \quad$ Atorvastatin $10 \mathrm{mg}$, fluvastatin $80 \mathrm{mg}$, lovastatin $40 \mathrm{mg}$ pitavastatin $2 \mathrm{mg}$, pravastatin $40 \mathrm{mg}$, rosuvastatin $5 \mathrm{mg}$, simvastatin $20 \mathrm{mg}$

$4 \quad$ Lovastatin $60 \mathrm{mg}$, simvastatin $30 \mathrm{mg}$

$5 \quad$ Atorvastatin $20 \mathrm{mg}$, pitavastatin $4 \mathrm{mg}$, pravastatin $80 \mathrm{mg}$, rosuvastatin $10 \mathrm{mg}$, simvastatin $40 \mathrm{mg}$

$6 \quad$ Atorvastatin $40 \mathrm{mg}$, rosuvastatin $20 \mathrm{mg}$, simvastatin $80 \mathrm{mg}$

$7 \quad$ Atorvastatin $80 \mathrm{mg}$, rosuvastatin $40 \mathrm{mg}$
ICD-9-CM (Equivalent ICD-10-CM)/HCPCS Codes

$82550,82552,82553,82554$

$359.4,359.89,359.9(\mathrm{G} 72.0, \mathrm{G} 72.2, \mathrm{G} 72.89, \mathrm{G} 72.9)$

729.1x (M60.9, M62.9, M79.1)

$564.0 \mathrm{x}(\mathrm{K} 59.0 \mathrm{x})$

$564.5,787.91$ (K59.1, R19.7)

782.1, 693.0, 995.1, 995.2, 695.1, 698.xx, 695.3 (R21, L27.0, L27.1, L27.8, L27.9, T78.3xx, T50.9xx, L51.x, L29.xx, L71.x

250.xx (E10.xxx, E11.xxx)

401.x-405.xx (I10-I16)

$7.7(\mathrm{E} 88.81)$

(6.

410.xx-414.xx (I20.x-I25.xxx)

$434 \mathrm{xl}, 435.9,436,437.1,437.9(\mathrm{I} 63 \mathrm{xxx}, 167.8 \mathrm{x}, \mathrm{I} 67.9)$

585.x (N18.x)

$278.02(\mathrm{E} 66.3)$

278.00, 278.01 (E66.0x, E66.1, E66.2, E66.8, E66.9)

$244.9(\mathrm{E} 03.8, \mathrm{E} 03.9)$
$728.88(\mathrm{M} 62.82)$ 